\title{
RELACIÓN ENTRE TRASTORNO DE CONDUCTA ALIMENTICIA Y ESTILO DE CRIANZA. Perspectiva de UN GRUPO DE ADultos Jóvenes de la CIUdAd de MedellíN
}

\author{
The Relationship Between Eating Disorders and \\ Upbringing. A Perspective from a Group of Young Adults \\ from Medellin City
}

\author{
Santiago Arroyave Pérez ${ }^{1}$, Anderson Gañán Moreno², \\ Andrés Miguel Vásquez OChoA ${ }^{3}$
}

https://doi.org/10.17533/udea.rp.e344170

\section{Resumen}

Se describe la perspectiva de un grupo de adultos jóvenes de la ciudad de Medellín sobre la relación de su trastorno de conducta alimenticia con el estilo de crianza que recibieron. Inicialmente, se plantea la influencia de diversos factores, desde el contexto social y familiar, en el proceso tanto de la crianza como de desarrollo de un TCA. Se realizó una investigación cualitativa con diseño descriptivo-transversal, para la cual se construyó una entrevista semiestructurada aplicada a cuatro adultos jóvenes de la ciudad de Medellín. Se encontró que los factores familiares y de crianza, junto con falencias en la comunicación y en el afecto, y el nivel de exigencia por parte de los padres, conlleva

Recibido: 2020-10-20 / Aceptado: 2021-01-29

Para citar este artículo en APA: Arroyave Pérez,

S., Gañán Moreno, A., y Vásquez Ochoa, A. M.

(2021). Relación entre trastorno de conducta alimenticia y estilo de crianza. Perspectiva de un grupo de adultos jóvenes de la ciudad de Medellín.

Revista de Psicología Universidad de Antioquia,

13(1), e344170. doi: https://doi.org/10.17533/ udea.rp.e344170 dificultades en la relación familiar y, a su vez, en el desarrollo de la autopercepción del sujeto. Las participantes son conscientes del trastorno y sus motivaciones, sin embargo, en su mayoría prefieren afrontarlo sin el apoyo familiar, dejando excluidos a los padres de los tratamientos. Por último, se plantea que existen diferentes factores sociales e interpersonales que contribuyen al desarrollo de un TCA; las participantes afirmaron que la crianza, la familia y el círculo social eran claves en este proceso.

Palabras clave: estilos de crianza, trastornos de conducta alimenticia, percepción, adultos jóvenes, familia.

Psicólogo de la Universidad EAfIT, Medellín, Colombia. Correo: sarroya3@eafit.edu.co; https://orcid.org/0000-0003-3835-7315.

2 Estudiante de Psicología y coordinador del Semillero de Investigación en Clínica del Trabajo y de las Organizaciones. Universidad EAFIT; https://orcid.org/0000-0001-5890-2187.

3 Candidato a Doctor en Humanidades, Universidad EAFIT. Docente del Departamento de Psicología de la misma universidad; https:// orcid.org/0000-0003-2403-3555. 


\begin{abstract}
The perspective of a group of young adults from the city of Medellín on the relationship of their eating disorder with the parenting style they received is described. Initially, the influence of various factors, from social and family context, in the process of both parenting and in the development of an ED was discussed. A qualitative research with a descriptive-transversal design was carried out, for which a semi-structured interview was constructed and applied to four young adults from the city of Medellín. It was found that family and upbringing factors, together with deficiencies in communication and affection, and the level of parental demands, lead to
\end{abstract}

difficulties in the family relationship and in turn with the development of the subject's self-perception. The participants are aware of the disorder and its motivations, however, most of them prefer to face it without family support, leaving the parents excluded from the treatments. Finally, it is suggested that there are different social and interpersonal factors that contribute to the development of an ED; the participants affirmed that upbringing, family and social circle were key in this process.

Keywords: parenting style, eating disorders, perception, young adulthood, family.

\title{
Introducción
}

La presente investigación busca describir la perspectiva de un grupo de adultos jóvenes de la ciudad de Medellín sobre la relación de su trastorno de conducta alimenticia (TСA) con el estilo de crianza que recibieron. Los TCA son manifestaciones extremas de la preocupación por el peso y la comida, que conllevan impulsos erráticos de alimentación; entre estos trastornos se encuentran la anorexia nerviosa, la bulimia nerviosa, la rumiación, etc. Estos pueden desarrollarse principalmente por una combinación de factores en el contexto inmediato, como los psicológicos (autoconcepto, autoimagen y autoestima), los interpersonales (problemas familiares) y los sociales (estereotipos) (American Psychiatric Association [APA], 2014).

Si bien en algunas descripciones se resalta una causalidad o explicación biológica, orgánica, como en la siguiente: «Los trastornos alimenticios se presentan cuando una persona no recibe la ingesta calórica que su cuerpo requiere para funcionar de acuerdo con su edad, estatura, ritmo de vida, etcétera» (Centro Nacional de Equidad de Género y Salud Reproductiva [CNEGSR], 2004, p. 11). Cabe mencionar que existen elementos psíquicos que también pueden tener influencia. 

grupo de adultos jóvenes de la ciudad de Medellín

Se encuentra una alta prevalencia global de Trastornos de la Conducta Alimentaria en la muestra analizada, que afecta en mayor grado a las mujeres. El factor de insatisfacción con la imagen corporal puede ser un elemento determinante, que podría inclinar a los adolescentes hacia conductas alimentarias y nutricionales inadecuadas (Barrera, Manrique y Ospina, 2012, p. 63).

En la actualidad tanto el contexto social como el interpersonal son grandes influencias en el desarrollo de TCA, como al igual lo son factores familiares y de crianza. Las relaciones de los primeros años de vida resultarán de importancia en el desarrollo de la autoimagen que cada sujeto tendrá a futuro, siendo, en algunos casos, una construcción generada a partir de los propios prejuicios de los padres o, incluso, del contexto en el que se encuentran los sujetos. Es aquí donde radica la relevancia de su crianza (National Institute of Mental Health, 2016). Aunque suele creerse socialmente que los TCA son exclusivos para las mujeres, esto dista de la realidad. Según Garrote y Palomares (2011), «12,46\% de las mujeres y un $24 \%$ de los hombres están obsesionados por la delgadez» (p. 106). Ahora bien, teniendo en cuenta que un porcentaje de casos, no poco significativo, se presenta en mujeres, esto podría constituir un criterio metodológico para la elección de la población participante de la investigación.

Por otro lado, es importante mencionar, como lo dicen Castillo, Carpintero y Sibaja (2015), que la interacción con los padres y la relación y comunicación afectiva hacen parte fundamental de la crianza. En este sentido, las bases éticas y morales de la familia determinarán el estilo de crianza, el cual influencia el desarrollo emocional y afectivo de los sujetos. Por ello se plantea que estos estilos son una base para formar personas que se adapten a las demandas sociales (Castillo et al., 2015). A partir de lo anterior, se puede plantear y explorar la relación entre la perspectiva que adultos jóvenes ofrecen sobre su crianza y sus propios TCA, en cuanto involucran recursos de afrontamiento.

En esta investigación se tendrán en cuenta los principales tipos de TCA: anorexia, bulimia, pica, evitación de la ingestión de alimentos y rumiación, los cuales han sido definidos previamente por la APA en el DSM-5 (2014) y serán descritos a continuación: 
La anorexia nerviosa se caracteriza por el miedo a engordar y tiene tres indicadores básicos: la restricción persistente de la ingesta de alimentos; el miedo intenso a ganar peso, o un comportamiento reiterativo que interfiere con el aumento de peso; y la alteración de la forma de percibir este último y la constitución propia. La bulimia consiste en ingerir una gran cantidad de alimentos, durante lo cual los individuos sienten una pérdida de control sobre la comida. Comúnmente esto conlleva sentir culpa y arrepentimiento tras la ingesta, por lo que el sujeto tratará de expulsar la comida manualmente, por medio del vómito.

La pica, por su parte, involucra ingerir persistentemente sustancias no nutritivas, aunque también algunas alimenticias, durante un período mínimo de un mes. Por otro lado, el trastorno de evitación de la ingestión de alimentos consiste en una falta de interés aparente por comer o alimentarse, ya sea por características organolépticas o desagradables de los alimentos. Por último, el trastorno de rumiación se caracteriza por regurgitar repetidamente alimentos durante un período mínimo de un mes.

Respecto a los estilos de crianza, en este estudio se consideran como factores importantes en la aparición de diversos TCA, dado que, justamente, el desarrollo psicológico de un sujeto se ve influenciado en gran medida por su crianza. Estos estilos consisten en los diferentes modos de reacción y respuesta de los padres a un estímulo o emoción del niño, a partir de lo cual harán que este aprenda a reaccionar y a adaptarse al contexto social de una manera determinada (Cajal, 2016).

Según Palacios (1999), existen cuatro estilos de crianza. Estos son:

Estilo democrático: es aquel en donde los padres mantienen muestras de afecto y aceptación explícitas, presentan sensibilidad hacia las necesidades de sus hijos y favorecen que se expresen verbalmente, exteriorizando sus sentimientos y sus pensamientos. Adicionalmente, dejan las normas claras haciéndoselas conocer y cumplen con los castigos o sanciones. La relación con sus hijos se reconoce como cálida, cercana, afectuosa y comunicativa. Utilizan reforzamientos positivos y los animan constantemente a superarse. En general, este estilo de crianza es el más buscado y recomendado, debido a que se pueden evidenciar efectos positivos para la salud mental de los hijos. Los niños 

grupo de adultos jóvenes de la ciudad de Medellín

que son educados con este estilo de crianza comúnmente se caracterizan por tener una autoestima alta y confianza en ellos mismos, afrontando así nuevas situaciones desde esa confianza y el entusiasmo.

Estilo autoritario: en él los padres dan gran importancia a las normas, el control y la exigencia, mientras que las emociones y los afectos no tienen un papel fundamental en las interacciones que se presentan con sus hijos. No se suele expresar abiertamente el carińo ni tampoco se presenta una sensibilidad o atención a los requerimientos de los hijos. Se exhibe una necesidad de control sobre ellos, la cual se usa en ocasiones como una reafirmación de poder. Se le resta importancia a que los niños comprendan el porqué de las cosas, simplemente se imponen. Tienden a utilizar los castigos y las amenazas como método para moldear la conducta de sus hijos, cumpliéndolos rigurosamente. Los hijos que son educados bajo este estilo tienden a presentar baja autoestima, debido a que sus padres no tenían en cuenta sus necesidades emocionales y afectivas.

Estilo permisivo: se caracteriza por altos niveles afectivos y emocionales. Los padres en este estilo de crianza priorizan el bienestar de su hijo ante cualquier cosa y son los intereses del niño los que gobiernan la dinámica familiar. Son padres poco exigentes, que plantean pocas normas y retos a sus hijos. Ante la dificultad permiten que estos desistan fácilmente y tenderán a no cumplir los castigos dados. Los hijos con padres permisivos se caracterizan por ser alegres, divertidos y expresivos, pero, al no estar acostumbrados a las normas, los límites, las exigencias y el esfuerzo, también son niños muy inmaduros, incapaces de controlar sus impulsos y que se rinden con facilidad.

Estilo indiferente/negligente: es un estilo de crianza en donde los padres prestan poca atención a sus hijos tanto en lo afectivo como en lo normativo. Las relaciones con ellos son frías y distantes, con poca sensibilidad respecto a sus necesidades. No establecen límites y normas, pero en ocasiones ejercen un control excesivo, injustificado y totalmente incoherente. Los hijos de padres con este estilo en ocasiones presentan problemas de identidad y baja autoestima, no conocen la importancia de la norma y por lo tanto difícilmente la cumplirán. Además, son poco sensibles a las necesidades de los demás y especialmente vulnerables a presentar problemas de conducta, con los conflictos personales y sociales que esto conlleva. 
Descrito lo anterior, se enfatiza, como fue planteado, que los diferentes estilos de crianza condicionan el desarrollo emocional y afectivo de los individuos (Castillo et al., 2015), y por lo tanto es posible que exista una relación entre ellos y los TCA. Por esto es importante considerar con detalle la influencia de estos estilos, puesto que las relaciones familiares son determinantes en el proceso de estructuración del sujeto, pues, por ejemplo, aspectos como la autoimagen se forman en los primeros años de vida.

Para la muestra poblacional de este estudio son de interés señalamientos sobre el contexto psicosocial, como los de Murillo y Pilar (2011), que advierten que el lugar y los acompañantes durante la ingesta de alimentos determinan, en adolescentes, el tipo y la calidad de estos; o los planteamientos de Ladero (2016), quien evidencia en su estudio, con estudiantes menores de 20 años, que las mujeres presentaron mayor obsesión por la delgadez e insatisfacción corporal, que los varones, mediante conductas bulímicas.

Finalmente, se puntualiza que la presente investigación pretende ampliar la comprensión de los TCA desde la perspectiva de los participantes, particularmente a partir de sus discursos y los modelos explicativos personales frente al surgimiento de sus propios trastornos y la relación con los estilos de crianza recibidos, pues las investigaciones suelen enfocarse en la cuantificación de factores similares, como la medición del riesgo de sufrir estos trastornos en la adolescencia o la cantidad de sujetos que los padecen en ámbitos universitarios. Por dicho motivo es que, se reitera, se busca dar a conocer la perspectiva de los sujetos.

\section{Método}

\section{Diseño de la investigación}

El enfoque de esta investigación es cualitativo, de tipo transversal descriptivo. En este abordaje se pretende profundizar en los significados construidos por sujetos acerca de sus propios TCA y los estilos de crianza recibidos, antes que cuantificar o medir correlaciones de variables. Es un proceder flexible que permite refinar y ajustar la investigación durante su ejecución. Igualmente, 
pretende conocer las situaciones, las costumbres y las actitudes predominantes a través de la descripción exacta de las actividades, los objetos, los procesos y las personas, relacionando dos o más categorías (van Dalen y Meyer, 1981).

\section{Instrumento}

La recolección de la información se realizó a través de entrevistas semiestructuradas, las cuales configuran un espacio diseñado para que se dé un encuentro entre personas, en el cual, a través del diálogo, se indagan aspectos relativos a representaciones, vivencias, experiencias, creencias del sujeto participante. Este modelo de entrevista permite que el entrevistador adapte su lenguaje de acuerdo con cada sujeto (Díaz, García, Hernández y Ruiz, 2013).

\section{Población}

Se contó con una muestra no probabilística de 4 adultos jóvenes, entre los 18 y 25 años, diagnosticados con algún TCA y residentes de la ciudad de Medellín, Colombia. Adicionalmente, se recolectó información sociodemográfica.

\section{Tabla 1}

Caracterización de los participantes

\begin{tabular}{lllllll}
\hline Sujeto & Edad & Sexo & Estado civil & Diagnósticos & Otros diagnósticos & Círculo familiar \\
\hline 1 & 20 & F & Soltera & Bulimia & No reporta & Madre \\
2 & 20 & F & Soltera & Anorexia & Ansiedad & Padre, madre y hermano \\
3 & 20 & F & Soltera & Anorexia & No reporta & Madre \\
4 & 20 & F & Soltera & Anorexia & Depresión, ansiedad & Padre y madre \\
\hline
\end{tabular}

\section{Procedimiento}

Se procedió con la recolección de información por medio de entrevistas semiestructuradas, con su respectivo protocolo; cada entrevista fue grabada y transcrita para su análisis. Los resultados obtenidos del análisis de las entrevistas fueron discutidos con los antecedentes y el marco de referencias conceptuales, 
lo que permitió lograr satisfactoriamente su interpretación. Además, se diseñó una matriz, la cual constaba de tres categorías principales: los estilos de crianza y la dinámica familiar en jóvenes con TCA; las afectaciones psicológicas de las personas que padecen TCA; y las posibles afectaciones de los estilos de crianza en el padecimiento de TCA.

Por último, las participantes firmaron el respectivo consentimiento informado, amparado en lo expuesto en la ley 1090 de 2006 y en la resolución 8430 de 1993 (artículo 11), cumpliendo con las consideraciones éticas de la investigación cualitativa.

\section{Resultados y discusión}

\section{Los estilos de crianza de jóvenes con trastornos de la conducta alimenticia}

Los estilos de crianza funcionan como la base para formar individuos que se adaptan a la sociedad, debido a que estos primeros se caracterizan por ser una expresión de la interacción, la comunicación, el afecto, los parámetros éticos y morales de la familia, para incidir en el desarrollo de los sujetos. Al respecto, a continuación se describen los resultados obtenidos sobre esta categoría.

Se ha planteado que la forma de comunicación de los padres condiciona en gran medida la estructuración de la personalidad, las habilidades y las competencias de sus hijos (Henao, Ramírez y Ramírez, 2007). Lo anterior se evidencia cuando el sujeto 1 menciona, refiriéndose a su madre, que: "Es difícil porque nunca hemos tenido buena relación, y las pocas veces como que hemos querido hablar sobre problemas que hay en la familia o en la casa todo termina mal, entonces es difícil". Además, el sujeto 3 expresa: "Con mi mamá ha sido con la persona con quien más problemitas he tenido, como que chocamos mucho en ciertos aspectos. Mi mamá es muy explosiva, muy llevada de su parecer", problemática que ha vivenciado desde su infancia hasta la actualidad. Así mismo, el sujeto 4 refiere: "En mi infancia corta mis dos papás trabajaban todo el día, todos los días, entonces yo nunca los veía muy seguido". Por ende, se puede inferir que la comunicación ha sido un factor que ha tenido falencias en el estilo de crianza de las participantes. 

grupo de adultos jóvenes de la ciudad de Medellín

Como ya se había planteado, en este caso Baumrind (1991) describe que las principales características del estilo de crianza autoritario son: la falta de comunicación y de afecto, la búsqueda desmedida de tener control sobre los hijos, y las críticas y exigencias de madurez en todo momento, remarcando un estilo ciertamente estricto y dominante. Respecto a la condición de afecto, el sujeto 1 mencionó: "Yo estoy como acostumbrada a que ella [la madre] sea más bien fría. [...] no es tanto de decirme que me quiere o no somos tan unidas [...] ambas somos como muy distantes". Así mismo, el sujeto 2 también comenta: "Mi mamá, de por sí, sí es cero cariñosa, es más como la típica mamá charra y seria; no le gusta mucho que le estén dando abrazos ni besos [...] nunca ha sido como de demostrar tanto como el afecto". Por su parte, el sujeto 3 expresa: "Mi mamá y yo no somos como tan cariñosas". De lo anterior se destacan las posibles falencias con relación a la falta de afecto vivida y narrada por los sujetos dentro de su hogar durante el proceso de la crianza, por parte de alguno de los miembros de su familia, especialmente la madre.

En lo concerniente a las características de este estilo en la dimensión normativa, los padres autoritarios son exigentes con la obediencia y la norma. Buscan que sus hijos se ajusten a un patrón de conducta que los lleva a crecer en medio de reglas establecidas, tanto morales como de comportamiento, las cuales deben ser cumplidas sin discusión, y, de no cumplirlas, se los castiga severamente. De acuerdo con lo anterior, el sujeto 1 expresa que "cuando era chiquita sí había más [reglas] y yo fui demasiado necia [...] a mí no me dejaban salir nunca, o sea, yo no llegué como a tener amiguitos en la cuadra, nunca salí a jugar". De igual modo, el sujeto 2 plantea: "Mi papá era muy estricto conmigo [a través de la norma], siempre me exigía mucho académicamente, entonces la parte del colegio fue muy fuerte. Mi mamá siempre estuvo ahí como defendiéndome, pero también exigía las calificaciones y pues nada”.

Al hablar del estilo de crianza autoritario, se entiende que este no facilita el diálogo, no tiene en cuenta las necesidades educativas, los intereses o las opiniones del niño (Baumrind, 1991). Sobre esto, el sujeto 1 indica: "Como a los 12 o 13 me quería pintar las uñas de negro y no me dejaban solo porque era negro, o me ponía cualquier camisa, por decirlo así, y si no les gustaba [a mis padres] me hacían cambiar y cosas de ese tipo". En cuanto el sujeto 2, este 
expresa: "Yo siento que cuando me pasan estas crisis yo las guardo, yo puedo contarles todo a mis papás, menos esto, porque siento que nunca me van a entender". En el caso del sujeto 3, este plantea: "Uno le lleva la contraria a mi mamá y no llega a ningún lado, con mi mamá no se puede hablar [...] yo no le puedo decir a ella mi punto de vista, ella siempre va a tener la razón”. Con esto podemos identificar que las restricciones de opinión o libertad afectan la toma de decisiones en la crianza de las participantes.

Además, Baumrind (1991) explica que el estilo autoritario es el que genera mayores repercusiones negativas sobre la socialización de los hijos. Crea una distancia entre estos y sus padres, provocando que los primeros sean menos cariñosos, más inseguros, descontentos e incluso retraídos. Pueden llegar a tener baja autoestima y no comprender por qué sus padres exigen ciertos comportamientos. En relación con estos efectos, el sujeto 1 manifiesta: "Como te dije que no tenía amigos, yo siempre me sentía como súper sola y como que sentía que no tenía papás [...], pero igual nuestra relación nunca como que mejoró, pues, la relación como que se estancó desde que yo era chiquita, también por muchas actitudes de ella [la madre] que yo sentía que me hacían como mucho dańo, entonces como que en ciertas partes le guardé mucho rencor y eso hasta el día de hoy se refleja". Por su parte, el sujeto 3 menciona: "Mi mamá antes era muy vanidosa con ella misma, muy enfocada como en su relación con esas parejas y yo de alguna forma me sentía como a un lado". Igualmente, el sujeto 4 declara: "Tuve como mi tiempo en el que yo decía: 'Yo no tengo papá. Yo no tuve como una infancia que uno diga como la más cercana a mis papás porque como te cuento los dos trabajaban mucho todo el día, entonces en la infancia no era como tan cercana a ninguno de los dos". Lo enunciado permite entender la percepción de las participantes frente a las consecuencias de los estilos de crianza de sus padres.

Por otro lado, Cuervo (2010) aclara que la familia influye en el desarrollo de conductas prosociales y la regulación de emociones, reforzando la idea de que es importante identificar la influencia de la familia y de los estilos y pautas de crianza en el desarrollo socioafectivo. Con lo que respecta al sujeto 1, este expone: "La relación con mi mamá siempre ha sido muy complicada [...] nunca hemos tenido buena relación". Por su parte, el sujeto 2 expresa: "Mi 

grupo de adultos jóvenes de la ciudad de Medellín

papá no es el que toma partido de ninguno de los dos, pero mi mamá sí quedó como con un bandito establecido con mi hermano [...] Como la relación y la comunicación estaba dividida en dos bandos, que era mi mamá y mi hermano, por un lado, y mi papá y yo, por otro, entonces creo que eso generó muchísimos problemas". En lo que respecta a los sujetos 3 y 4, tal como se declara en los testimonios inmediatamente anteriores, refieren una relación distante con sus padres. Esto nos permite dar cuenta de diversas situaciones que señalan dificultades relacionales, desde la perspectiva de los sujetos con relación a su crianza, la cual presenta características autoritarias.

En síntesis, desde la perspectiva de las participantes es posible dar cuenta de diversos aspectos familiares y de crianza que presentan falencias o dificultades, tales como: la comunicación, el afecto, el nivel de exigencia y la libre expresión; factores desde los cuales se reconoce un claro deterioro en la relación entre padres e hijas, dadas estas diversas muestras de conflictos y malestar.

\section{Afectaciones psicológicas de personas que padecen trastornos de conducta alimenticia}

Como se expresó anteriormente, los TCA se caracterizan por alteraciones en los patrones alimentarios, como consecuencia de la preocupación excesiva por el peso, la imagen corporal y la relación con la comida, conllevando grandes afectaciones anímicas en quienes los presentan. A continuación, se describen los resultados de esta categoría a partir de las entrevistas de las cuatro participantes.

Se recuerda que según el CNEGSR (2004), los trastornos alimenticios se presentan cuando una persona no recibe la ingesta calórica que su cuerpo requiere para funcionar de acuerdo con su edad, estatura, ritmo de vida, etcétera. Sobre esta condición, el sujeto 1 expresa: "Llevaba un año sin comer y vomitando". Por su parte, el sujeto 2 refiere: "Yo estaba sufriendo anorexia y eso era una anorexia nerviosa porque yo ya no me acordaba si comía o no comía, y me daban ataques de ansiedad, y yo temblaba y lloraba a puerta cerrada y callada, porque nadie se tenía que enterar de eso”. Así mismo, el sujeto 3 declara: "tuve anorexia por el tema de dejar de comer; anorexia 
nerviosa porque uno le coge miedo a ciertos alimentos". Por último, el sujeto 4 señala: "Bulimia, si no estoy mal, fue hace dos años [según autodiagnóstico], y la anorexia creo que un año larguito [...] busqué alternativas para conseguir o verme en ese sentido de la manera en la que yo quería”. De este modo, las participantes dejan claro que padecen o padecieron un TCA.

Adicionalmente, el CNEGSR (2004) manifiesta que la alimentación trastornada constituye, para quienes la presentan, formas de nutrirse o protegerse, de expresar ira, de autocastigarse, de apaciguar tensiones insoportables, de distanciarse de situaciones dolorosas y hasta una manera de crear una identidad. Al respecto, el sujeto 1 menciona: "Es muy duro porque yo pensaba que lo que estaba haciendo estaba bien, porque yo me veía o me pesaba y estaba como quería estar, pero cuando te dicen que no estás [bien] es como un choque muy fuerte". En cambio, el sujeto 3 declara: "Me empecé a desvalorizar yo misma". En su caso, el sujeto 4 comenta que también se desvalorizaba en los momentos en los que era invitada a eventos con alimentación a base de grasa saturada: "Yo me ataco muchísimo, porque no tengo esa vocecita constante diciéndome: 'Estás gorda', 'Tienes que rebajar', 'No vas a ir a tal plan', porque me pasaba mucho eso, me invitaban a comer pizza con las amigas... yo llegué al punto de rechazar salidas porque sabía que íbamos a comer mal". Con lo anterior, podemos identificar en la perspectiva de las participantes que desde el inicio de sus trastornos reconocen diversos cambios en factores comportamentales, anímicos, sociales, de autorrepresentación, entre otros, destacando que para cada una se presentaron modificaciones y afectaciones diferentes.

«El tratamiento de un trastorno de la alimentación depende del trastorno en particular y [s] us síntomas. Por lo general, comprende una combinación de terapia psicológica, educación sobre alimentación, supervisión médica y, algunas veces, medicamentos» (Mayo Clinic, 2007, párr. 1). Aun las posibilidades de tratamiento, siempre hay un grado de abandono o simplemente los pacientes cuentan con una falta total de conciencia de riesgo sobre el trastorno, o se niegan a buscar ayuda (Mayo Clinic, 2007). En relación con la atención a su trastorno, el sujeto 1 comenta: "Conocí a una persona que también tenía bulimia y me ayudó mucho porque ella sí iba de la mano 

grupo de adultos jóvenes de la ciudad de Medellín

con un psiquiatra; había estado en rehabilitación. Ella fue la que me ayudó en mayor parte". El sujeto 3, sobre su caso, expresa: "Yo siempre quiero resolver todo sola, no quiero molestar a nadie, no quiero hablar con nadie”. De otro lado, el sujeto 4 narra: "Básicamente me obligaban a quitarme las pastillas con las que empecé a dejar de comer, pero yo hoy en día todavía me las tomo, no hay un día que no me las tome; ya no es lo mismo, porque sí, uno antes estaba lleno todo el día”. Con base en lo anterior, se logra dar cuenta de que los métodos utilizados por las participantes, tanto en relación con la expresión de los síntomas como en la búsqueda de soluciones para estos, varían según su contexto. Aun así, es de vital importancia resaltar, en general, la falta de consciencia de gravedad del trastorno, de adherencia a los posibles tratamientos, y de acompañamiento por parte de familiares y profesionales, lo cual lleva, por ende, a que no se logren obtener los beneficios del proceso terapéutico o mejoras de los síntomas.

Es característico de las personas con trastornos alimenticios aislarse socialmente, aunque recurren a personas de confianza (mejores amigos, pareja) en busca de aseguramiento y validación emocional, sin dejar de lado una permanente preocupación por el peso y la delgadez (CNEGSR, 2004). También es común que estén irritables, malhumoradas o se sientan culpables. Presentan síntomas como la ansiedad, la impulsividad, la inestabilidad y la distorsión de la percepción de sí mismo. Sobre su vivencia del trastorno, el sujeto 1 expresa que: "Cuando me daban atracones sentía que era la peor persona y me daban pensamientos suicidas, me culpaba por tener antojos, como que sentía que la comida era mi enemiga y yo era mi enemiga también, entonces estaba demasiado mal". De la misma manera, el sujeto 2 refiere: "A nivel emocional me afectó muchísimo porque es como si vos a toda hora después de todo eso que te pasó tuvieras una voz en tu cabeza que te dijera: 'Ay no, estás demasiado gorda; tienes que hacer más ejercicio; tienes que comer menos; vomita, mira, tienes el baño para vomitar; tienes que hacer todo lo posible para estar igual de flaca que esta vieja; pero mira todas las modelos que han salido adelante; que no te venga la menstruación no es importante"”. Respecto a su caso, el sujeto 3 menciona que: "Empecé como yo a mirarme todos los días al espejo y siempre quería como más, como verme más flaca, entonces dejaba de comer 
más [...] yo le tenía miedo al arroz, a las papitas, a los carbohidratos y a los dulces". Por otra parte, el sujeto 4 aclara que su trastorno le generó "problemas de autoestima y de inseguridades". A partir de lo anterior, las afectaciones anímicas en las participantes fueron evidenciadas; estas se presentaron de diferentes formas, como el aislamiento, los problemas de autoestima, etc.

El CNEGSR (2004) sugiere que la vivencia subjetiva, de acuerdo con la historia personal, es central en este tipo de padecimientos. El sujeto 1 plantea: "Siempre intento como estar bien para las otras personas porque no me gusta que me vean triste". Por su parte, el sujeto 4 apunta: "Yo considero que soy extrovertida en la mayoría del tiempo, [pero también] soy más bien calladita, soy seria [...] Sí me he considerado una persona triste, o sea, una persona con una vida triste con momentos felices". Con base en estas valoraciones de dos de las participantes, se entiende que tienen una variada autopercepción emocional, debido a su contexto y su historia personal.

En conclusión, existe una consciencia del trastorno por parte de las participantes, al igual que de las motivaciones para tener esas conductas. Ahora, en relación con los tratamientos de sus trastornos, estas indican que prefirieron afrontarlos de manera individual, sin apoyo de sus padres y dejándolos por fuera de cualquier aspecto de este proceso, como lo menciona el sujeto 3: "Yo nunca les conté a mis papás, ellos nunca pensaron que era un trastorno, sino que pensaron que era lo del pie. Nunca hablé con nadie, nunca le conté a nadie; nunca, cero, nada”. Sin embargo, se encontró un alto nivel de consecuencias negativas y secuelas anímicas, debido a esta reserva, como culpa, baja autoestima, depresión, aislamiento, entre otros.

\section{Posibles efectos de los estilos de crianza en el padecimiento de los trastornos alimenticios}

Los estilos de crianza pueden facilitar el desarrollo de TCA, debido a que implican diferentes creencias familiares, características, comportamientos capaces de afectar las habilidades de afrontamiento que influyen en la vida y crecimiento de los hijos y su estabilidad emocional. Bajo esta consideración, ahora se presentarán los resultados obtenidos en esta categoría. 

grupo de adultos jóvenes de la ciudad de Medellín

Uno de los posibles efectos de los estilos de crianza en el padecimiento de los trastornos alimenticios se basa en el perfeccionismo, que representa un rasgo multidimensional caracterizado por una elevada autoexigencia y una autoevaluación excesivamente crítica (Bartolomé, 2016). Al respecto, el sujeto 1 señala: "Como que yo siempre sentía que no era lo suficiente para el resto de las personas [...] sentía que no era suficiente". En cuanto al sujeto 3, este propone, refiriéndose al inicio del trastorno: "Fue digamos el círculo social de las amistades y digamos esos comentarios de mi tía que también influyeron, porque era como comparándome con mis primas y yo obviamente al ver a mis primas más flacas”. Por su parte, el sujeto 4 comenta, también con relación al inicio de su trastorno: "En parte puede ser porque mi mamá también hacía así, de esforzarse ella misma con las pastillas, con las dietas, y en parte porque yo estuve la mitad de mi vida en un colegio femenino y eso siempre había una competencia brutal en el que cualquier cosa es una crítica, cualquier cosa es la bajada de autoestima". Entonces, podemos dar cuenta del rasgo perfeccionista en 3 de las 4 participantes; dicho rasgo va de la mano con el estilo de crianza autoritario, el cual se basa en la alta exigencia.

Por otra parte, Mateos-Agut et al. (2014) mencionan que en familias con hijos con TCA las expectativas paternas suelen ser polarizadas, bien por ser exigentes, poco realistas y con una marcada sobreprotección, o bien, despreocupadas y con menor control de la conducta de sus hijos. Así pues, el sujeto 1 expresa: "Yo eso [el origen del trastorno] sí lo he analizado mucho, mucho, y pienso que de pronto fue como crecer con una mamá que me exigía todo el tiempo a la margen como perfecta [...] Yo llevaba un examen en 4.8 y me regañaba, me decía: '¿Por qué no tienes un 5.0?’, entonces yo siempre quería ser perfecta en todos los aspectos. En haber crecido también como sola, de alguna manera”. De lo expresado por el sujeto 3, se retoma el fragmento anterior: "Digamos esos comentarios de mi tía que también influyeron, porque era como comparándome con mis primas y yo obviamente al ver a mis primas más flacas". En su caso, sobre este respecto el sujeto 4 cuenta con una experiencia similar: "En mi familia yo creo que absolutamente todas las mujeres son preocupadas por su peso, por su aspecto, siempre son preguntándose si se ven gordas, si les ve bien esto, si se ven cachetonas, entonces creo que 
también es algo de educación; yo aprendí a ver eso y dije como que eso debe ser normal, pero que yo diga que me educaron para sentirme así, no, yo creo que es algo adquirido". Con lo anterior, se puede entender que en la crianza de las participantes se evidencia exigencia, perfeccionismo o despreocupación; además, se remarca que en su discurso mencionan vivencias solitarias, sin apoyo de los padres o interés de su parte.

El CNEGSR (2004) aclara que las causales de los trastornos alimenticios son multifactoriales y emergen en ambientes diversos de manera procesal, en un momento evolutivo particular. En consonancia con lo anterior, el sujeto 1 menciona, refiriéndose a su madre: "Haber crecido también como sola [...] Siempre era como haciéndome arrepentir de una decisión de ponerme un jean muy pegado, por ejemplo, o me decía como que tenía que empezar a comer menos y hacer más ejercicio, y siempre me recalcaba eso, entonces eso se quedó en mi cabeza”. Por otra parte, se vuelve sobre la siguiente declaración del sujeto 4: "Porque yo estuve la mitad de mi vida en un colegio femenino y eso siempre había una competencia brutal en el que cualquier cosa es una crítica, cualquier cosa es la bajada de autoestima”. Por lo tanto, desde la perspectiva de las participantes se presentan factores familiares, sociales y de crianza, que llegan a dar pie a la formación de un TCA.

«Las personas que sufren un TCA basan su autoestima en su imagen corporal y piensan que estando más delgadas se sentirán mejor y, sobre todo, conseguirán la aceptación de los demás y de ellas mismas» (Díaz, 2017, párr. 3). Sobre la autoestima, el sujeto 1 expresa: "Yo diría que es una montańa rusa, es bien inestable; hay días en los que me siento bien, tranquila, y hay otros días en los que tapo los espejos porque no me quiero ver, o no me gusta salir porque no me siento bien para salir". El sujeto 2 menciona: "Yo esa seguridad ya no la tengo y la autoestima que yo tengo en este momento a veces puede crecer como a veces puede bajar"; y el sujeto 4 dice: "La he trabajado casi que toda mi vida, porque antes sí era muy bajita”. En articulación con las situaciones de exigencia social y familiar planteadas anteriormente, se puede dar cuenta de que la autoestima ha sido un factor de relevancia para las participantes, de cara a sus TCA. Situaciones propiciadas por sus mismas familias respecto a tener una fijación por la imagen corporal, y los imaginarios de perfección 

grupo de adultos jóvenes de la ciudad de Medellín

de las participantes, impulsan la sintomatología del trastorno, puesto que en algunos casos dejar de comer se concibe como un medio para obtener el cuerpo deseado o necesario, para el sujeto o su contexto

Según mencionan Mateos-Agut et al. (2014), se ha reportado que entre familias de los pacientes con TCA el liderazgo es difuso y los vínculos entre padres e hijos suelen ser ambivalentes y poco afectuosos. Comenta el sujeto 1: "Mi mamá es la persona que me hace sentir así [alude a un sentimiento de tristeza y humillación], si se supone que es la persona que me tiene que hacer sentir como amada. [...] Mi mamá fue súper dura porque ella me culpaba a mí, decía que eso ni siquiera era un trastorno, sino como una exageración, una bobada de adolescencia”. Por otro lado, el sujeto 3 expresa, como se presentó previamente: "Yo nunca les conté a mis papás, ellos nunca pensaron que era un trastorno, sino que pensaron que era lo del pie; nunca hablé con nadie". Respecto a su caso y el apoyo que recibió, el sujeto 4 refiere: "Fue más que todo en parte del psiquiatra, porque yo en este momento... mi mamá no tiene idea de que estoy aquí contigo, porque ella no sabe de este trastorno. Nadie. Solamente como mis amigos más cercanos, porque en mi casa lo ven más como una bobada, como un capricho. Yo me recosté más que todo en mis amigos y mi psiquiatra, porque yo les decía: 'Yo no quiero comer, yo no tengo ganas, yo sigo con los medicamentos que me mantienen llena y ya, no necesito nada más en mi vida”. Se puede identificar que la influencia del TCA afecta las relaciones familiares en adultos jóvenes, por la naturalización de eventos y por la falta de comunicación familiar.

En síntesis, se evidencia que existen diferentes factores sociales e interpersonales que intervienen en la formación del TCA; ahora bien, las participantes confirman que la crianza es uno de esos factores clave, al igual que la familia y el círculo social. Por otra parte, la autopercepción de insuficiencia, la soledad y la falta de afecto son otros factores que tienen un notable peso en la constitución de dicho trastorno. 


\section{Conclusiones}

Esta investigación pretendió describir la perspectiva de un grupo de adultos jóvenes de la ciudad de Medellín sobre la relación de su TCA con el estilo de crianza que recibieron. Principalmente se concluye, de acuerdo con los estilos de crianza del grupo de jóvenes con trastornos alimenticios entrevistados, que estos, junto con el ambiente familiar, son fundamentales para el aprendizaje, el desarrollo social, intrapersonal y emocional, puesto que las participantes afirman que su crianza ha perjudicado o favorecido aspectos de sus vidas.

No es posible afirmar que uno de los estilos de crianza se relaciona en su totalidad con los TCA; sin embargo, según los resultados de las entrevistas realizadas, se rescata que las características de la crianza que recibieron las participantes apuntan al estilo autoritario, debido a que aspectos como las muestras de afecto, la buena comunicación, la libertad de expresión, las normas, la exigencia y el control, y la atención brindada por los padres, presentan dificultades o falencias significativas desde el punto de vista de las entrevistadas. Este estilo puede tener como consecuencias una relación complicada entre los miembros del núcleo familiar, y en lo que concierne al hijo, problemas emocionales, baja autoestima y poca autoconfianza.

Con relación a las afectaciones psicológicas de las personas que padecen TCA, se corrobora, con base en lo descrito por las participantes, que estos se desarrollan debido a la excesiva preocupación por el peso y la imagen corporal, mayormente en el periodo de la adolescencia. Sin embargo, también es posible encontrar casos en la adultez. Esto genera un sinfín de afectaciones y secuelas anímicas significativas, como baja autoestima, miedo a ciertos alimentos, aislamiento social, depresión, culpa, ansiedad, entre otras.

Es posible observar que el grupo de personas entrevistadas cargan con el peso de los estereotipos sociales y culturales respecto al imperativo de un cuerpo delgado o fitness, incluso si para ello se recurre a procedimientos estéticos. Cierto es que esto provoca oleadas de inseguridades e insatisfacción constante sobre su imagen corporal, además de poca aceptación hacia sí mismas, gracias a la cantidad de comparaciones que realizan entre ellas y modelos, figuras públicas o, incluso, personas de su propia familia y su círculo 

grupo de adultos jóvenes de la ciudad de Medellín

social. Teniendo en cuenta lo anterior, y que los contextos familiares y sociales de estas participantes propician un ambiente de comparación, estas llegan a sentir una presión continua frente a la figura corporal. Muchas veces la familia es la que más puede incidir en la formación de los trastornos alimenticios mediante comentarios que resultan inoportunos sobre la comida, el peso, la talla o la imagen corporal en general.

La inseguridad toma gran relevancia en la vida de los sujetos con TCA, puesto que se vuelve parte de su cotidianidad, siendo acompañada habitualmente de un sentimiento de insuficiencia. En el caso de las participantes, dichas inseguridades respecto a su peso o imagen corporal se fundaron a raíz de sus relaciones interpersonales en el contexto social y familiar. Así mismo, la ansiedad a raíz de un TCA afecta diferentes aspectos de la vida de un sujeto; la comida y la vida social pasan a ser un tema de conflicto, causante de ansiedad.

Para finalizar, parte de la inseguridad y la ansiedad ocasionadas por los criterios estéticos impuestos por las personas del círculo social de las participantes podrían propiciar diversas secuelas psicológicas, modificando muchas de sus conductas referentes a la comida, la autopercepción y las relaciones sociales.

Con respecto a las afectaciones generadas por los estilos de crianza en el padecimiento de TCA, podemos dar cuenta que estos estilos son fundamentales en el desarrollo del sujeto tanto de forma positiva como negativa: favorecen la autoestima alta y la confianza en sí mismo, o bien, la baja autoestima; impactan la alegría, la expresividad, la inmadurez, el control de impulsos, la importancia de la normas, entre otras características, debido a que condicionan el desarrollo emocional y social de los jóvenes en crecimiento.

Se logra evidenciar un marcado estilo de crianza autoritario en las dinámicas familiares de sujetos con TCA, el cual se caracteriza, como ha sido descrito a lo largo del texto, por otorgar una gran importancia a las normas, el control y la exigencia, así como por la falta de afecto y poca comunicación con los hijos, dejando por fuera la posibilidad de estos de opinar por la notable autoridad que ejercen los padres. El alto nivel de exigencia en el estilo autoritario conlleva que los sujetos criados bajo este presenten un alto grado de perfeccionismo en su vida, además de que tengan una marcada sensación de insuficiencia 
en diversos ámbitos, llevando a dificultades en la relación con los padres y a dificultades emocionales, como ansiedad y otras afectaciones psicológicas.

No obstante, cabe aclarar nuevamente que otros factores como la familia, el círculo y contexto social, la autopercepción, el sentimiento de soledad, etc., son también relevantes e influyentes en el desarrollo de un TCA.

A continuación, se señalan los principales aportes de la investigación. Esta constituye una forma de darle visibilidad a los trastornos alimenticios desde la perspectiva de quienes los padecen, específicamente, reconociendo qué factores consideran que los detonan. Así mismo, comprende una orientación hacia la intervención terapéutica, en la medida que permite dar cuenta de elementos de la vida de un sujeto con TCA que pueden ayudar a mejorar los procesos y métodos para su tratamiento. Por otra parte, ofrece un aporte académico en relación con la comprensión de componentes clave en el desarrollo de dichos trastornos. En ese sentido, la investigación presenta congruencias entre diversas características del estilo de crianza autoritario y las de los TCA, como también permite dar pie a procesos de aprendizaje sobre los estilos de crianza y su importancia y gran influencia en diversos factores de la vida de una persona durante su desarrollo.

Con relación a las limitaciones de la investigación, se evidencia que al estar la muestra totalmente conformada por mujeres, conduce a que el análisis de la relación entre el estilo de crianza recibido y los trastornos de conducta alimenticia solo se dé desde un género específico, aunque este sea el más afectado por estos trastornos; lo anterior deja la ventana abierta para realizar la investigación en una muestra conformada por ambos géneros, o totalmente masculina, para así realizar cuadros comparativos de investigación.

\section{Referencias}

American Psychiatric Association (2014). Manual diagnóstico y estadístico de los trastornos mentales. DSM-5. Bogotá: Médica Panamericana.

Barrera, L., Manrique, F., y Ospina, J. (2012). Evaluación de la conducta alimentaria en estudiantes de Tunja (Boyacá, Colombia). Hacía promoc. salud, 18(2), 55-65. Recuperado de https://bit.ly/3iuHZaW 
Relación entre trastorno de conducta alimenticia y estilo de crianza. Perspectiva de un grupo de adultos jóvenes de la ciudad de Medellín

Baumrind, D. (1991). Parenting Styles and Adolescent Development. En J. Brooks Gunn, R. Lerner, y A. C. Petersen (Eds.), The Encyclopedia of Adolescence (pp. 746-758). New York: Garland.

Bartolomé, C. (2016). Emociones y trastornos de la conducta alimentaria: correlatos clinicos y abordajes terapéuticos basados en nuevas tecnologías (Tesis doctoral). Universitat de Barcelona. Facultat de Medicina, Barcelona.

Cajal, A. (2016). Los 4 estilos de crianza educativos y sus características. Lifeder. https:// www.lifeder.com/estilos-crianza/

Castillo, S., Carpintero, K., y Sibaja, L. (2015). Estudio de los estilos de crianza en niños, niñas y adolescentes en Colombia: un estado del arte. Revista Búsqueda, 2(15) 64-71. doi: https://doi.org/10.21892/01239813.97

Centro Nacional de Equidad de Género y Salud Reproductiva. (2004). Guía de Trastornos alimenticios. México: Secretaría de Salud. Recuperado de https://bit. ly/2KClwfI

Cuervo, Á. (2010). Pautas de crianza y desarrollo socioafectivo en la infancia. Revista Diversitas, 6(1), 111-121. Recuperado de https://bit.ly/39RT1TS

Dalen, D. van y Meyer, W. (1981). Estrategia de la investigación descriptiva. En Manual de técnica de la investigación educacional. Barcelona: Paidós.

Díaz, L., García, U., Hernández, M., y Ruiz, M. (2013). La entrevista, recurso flexible y dinámico. Investigación en Educación Médica, 2(7). Recuperado de https://bit. ly/3gZaBud

Díaz, V. (2017). ¿Cómo influye la autoestima en los trastornos de alimentación (TCA)? Grupo XxI Psicología. https://www.grupoxxi-psicologia.net/blog/influye-laautoestima-los-trastornos-alimentacion/

Garrote, D. y Palomares, A. (2011). Diferencias de género en la incidencia de los trastornos de la conducta alimentaria (тСA) en los factores de personalidad del alumnado de educación secundaria. Enseñanza \& Teaching, 29(1), 95-109. Recuperado de https://bit.ly/3iuIWQH

Henao, G., Ramírez, C., y Ramírez, L. (2007). Las prácticas educativas familiares como facilitadoras del proceso de desarrollo en el niño y niña. El Ágora USB, 7(2), 233-240. doi: https://doi.org/10.21500/16578031.1646

Ladero, M. (2016). Influencia de los estereotipos de belleza actuales en la percepción de la imagen corporal (Tesis de pregrado). Universidad Pontificia de Salamanca, España. 
Mateos-Agut, M., García-Alonso, I., Gándara-Martín, J. J. de la, Vegas-Miguel, M. I., Sebastián-Vega, C., Sanz-Cid, B., Martínez-Villares, A., y Martín-Martínez, E. (2014). Family Structure and Eating Behavior Disorders. Actas españolas de psiquiatría, 42(6), 267-280. https://bit.ly/35X81NI

Mayo Clinic. (2017). Tratamiento para un trastorno de la alimentación: infórmate sobre tus opciones. https://mayocl.in/35YWpLE

National Institute of Mental Health (2016). Los trastornos de la alimentación: un problema que va más allá de la comida. https://bit.ly/3kfdfea

Murillo, O. y Pilar, M. (2011). La alimentación de los adolescentes: el lugar y la compañía determinan las prácticas alimentarias. AQUICHAN, 11(2), 199-216. Recuperado de https://bit.ly/3ai0NX7

Palacios, J. (1999). La familia y su papel en el desarrollo afectivo y social. En I. Etxebarría, M. J. Fuentes, F. López y M. J. Ortiz (coords.). Desarrollo afectivo y social (pp. 267-284). Madrid: Pirámide. 